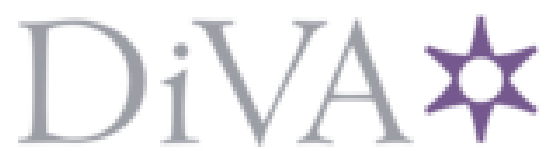

http://www.diva-portal.org

\title{
Postprint
}

This is the accepted version of a paper presented at European Conference on Software Architecture, Canterbury, UK, 11-15 September, 2017.

Citation for the original published paper:

Olsson, T., Ericsson, M., Wingkvist, A. (2017)

The relationship of code churn and architectural violations in the open source software JabRef.

In: ECSA '17 Proceedings of the 11th European Conference on Software Architecture:

Companion Proceedings (pp. 152-158). New York, NY, USA: ACM Publications

ACM International Conference Proceeding Series

https://doi.org/10.1145/3129790.3129810

N.B. When citing this work, cite the original published paper.

"(C) $\{$ Owner/Author $\mid$ ACM $\}\{2017\}$. This is the author's version of the work. It is posted here for your personal use. Not for redistribution. The definitive Version of Record was published in \{Proceedings of the 11th European Conference on Software Architecture: Companion Proceedings $\}$, http:// dx.doi.org/10.1145//3129790.3129810"

Permanent link to this version:

http://urn.kb.se/resolve?urn=urn:nbn:se:Inu:diva-68408 


\section{The Relationship of Code Churn and Architectural Violations in the Open Source Software JabRef}

\author{
Tobias Olsson \\ Department of Computer Science \\ Linnaeus University \\ Kalmar, Sweden \\ tobias.olsson@lnu.se
}

\author{
Morgan Ericsson \\ Department of Computer Science \\ Linnaeus University \\ Växjö, Sweden \\ morgan.ericsson@lnu.se
}

\author{
Anna Wingkvist \\ Department of Computer Science \\ Linnaeus University \\ Växjö, Sweden \\ anna.wingkvist@lnu.se
}

\begin{abstract}
The open source application JabRef has been developed since 2003. In 2015, the developers decided to make an architectural refactoring as continued development was deemed too demanding. The developers also introduced Static Architecture Conformance Checking (SACC) to prevent violations to the intended architecture. Measurements mined from source code repositories such as code churn and code ownership has been linked to several problems, for example fault proneness, security vulnerabilities, code smells, and degraded maintainability. The root cause of such problems can be architectural. To determine the impact of the refactoring of JabRef, we measure the code churn and code ownership before and after the refactoring and find that large files with violations had a significantly higher code churn than large files without violations before the refactoring. After the refactoring, the files that had violations show a more normal code churn. We find no such effect on code ownership. We conclude that files that contain violations detectable by SACC methods are connected to higher than normal code churn.
\end{abstract}

\section{CCS CONCEPTS}

-Software and its engineering $\rightarrow$ Software architectures; Software verification and validation;

\section{KEYWORDS}

Software Architecture, Conformance Checking, Repository Data Mining

\section{ACM Reference format:}

Tobias Olsson, Morgan Ericsson, and Anna Wingkvist. 2017. The Relationship of Code Churn and Architectural Violations in the Open Source Software JabRef. In Proceedings of European Conference on Software Architecture, Canterbury, UK, Sep 2017 (SAEroCon), 7 pages.

https://doi.org/10.475/123_4

\section{INTRODUCTION}

JabRef $^{1}$ is a Java Open Source Software (OSS) desktop application for managing bibliographic references. The first version of JabRef

\footnotetext{
${ }^{1}$ https://www.jabref.org

Permission to make digital or hard copies of part or all of this work for personal or classroom use is granted without fee provided that copies are not made or distributed for profit or commercial advantage and that copies bear this notice and the full citation on the first page. Copyrights for third-party components of this work must be honored.

For all other uses, contact the owner/author(s).

SAEroCon, Sep 2017, Canterbury, UK

(C) 2017 Copyright held by the owner/author(s)

ACM ISBN 123-4567-24-567/08/06 .. \$15.00

https://doi.org/10.475/123_4
}

was released in 2003 and it has since then been under active development; the current version was released in early 2017. JabRef seems to have lacked an explicit software architecture for the majority of its development. In 2015, the developers deemed that further development was too hard so a new software architecture was introduced. During the following releases, more and more of the Java classes in the application were refactored into this new architecture. To facilitate the transition, automated unit tests that analyze the source code were introduced to ensure that the intended structure of packages in the architecture was not violated. These unit tests is one of many possible ways to perform Static Architectural Conformance Checking (SACC) [4, 16, 18, 23, 24, 29, 30, 34].

Detection methods for architectural violations is a well-established area of research, but we find that there is a lack of research results that focus on the analysis and tangible impact of architectural violations as well as the effect of refactoring such violations [1, 15]. Source code repository mining provides the opportunity to understand how software evolves, which developers are involved, and what changes are made to the source code of a system [14, 19, 39]. We can use mining to study the effects of refactoring architectural violations by studying changes to source code size (code churn) as well as the contributions of different developers (code ownership). When code churn and code ownership deviates from what can be observed during "normal evolution", it might indicate that there are problems [10, 27].

JabRef provides an opportunity for us to study code churn and code ownership in the light of architectural violations in a project that use SACC. Our overall aim is to determine whether there is a difference in code churn and ownership between files that do and do not contain architectural violations as well as how architectural refactoring impacts files with violations. Is there a link between architectural violations and code churn or code ownership? We seek to answer the following research questions:

RQ1. Do files with architectural violations have a difference in code churn compared to files without architectural violations?

RQ2. Do files that were refactored to remove architectural violations have a difference in code churn compared to files without architectural violations?

RQ3. Do files with architectural violations have a difference in code ownership compared to files without architectural violations?

RQ4. Do files that were refactored to remove architectural violations have a difference in code churn compared to files without architectural violations? 


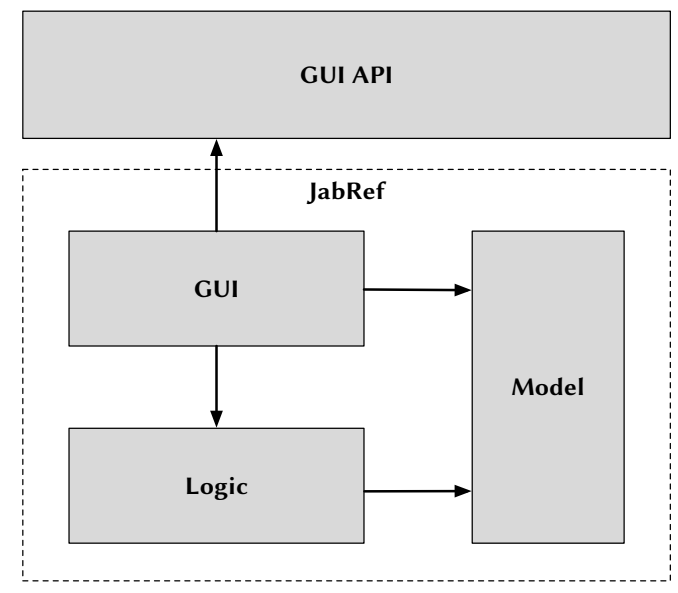

Figure 1: The intended architecture components and relations in JabRef.

We aim to contribute to fill the research gap by studying tangible effects of using SACC, as well as to understand if there is a link between code churn, code ownership, and architectural violations in the situations where a software architecture was introduced during the development and the source code was subsequently refactorerd to conform to this architecture.

\section{BACKGROUND}

During the evolution of a software system, problems that can inhibit a system's architectural qualities, such as performance, security, reusability, and maintainability arise [20, 21, 35]. These problems are for example caused by a lack of explicit architecture, mistakes in the implementation, new architectural requirements, or new technologies $[6,17,36]$. They can, in themselves be minor, but the long-time accumulation can inhibit the qualities. We refer instances of these problems in the source code as architectural violations. One way to avoid these architectural violations in the source code is to use SACC tools. These tools identify where the source code breaks an intended architectural constraint and alert developers to the existence and location $[22,28]$. They often rely on dependencies embedded in source code statements, and compare these with the desired and allowed dependencies in the software architecture. Methods such as Reflexion Models [4, 16, 23, 24], Dependency Rules [29, 30, 34], and Dependency Structure Matrices [18] have been used to find and visualize architectural violations.

JabRef introduced SACC to enforce the new architecture following a major refactoring in 2015. The new intended architecture consists of three layers: Model, Logic, and GUI. A fourth component outside the application itself is the GUI API (e.g., Java Swing and Abstract Window Toolkit (AWT)).

JabRef uses a simple approach to SACC that is based on unit tests that scan source code files and look at import statements. The architectural tests implement the following rules:

(1) Model does not import from GUI, Logic, or the GUI API

(2) Logic does not import from GUI, or the GUI API
Hall and Munson introduce the concept of code churn and code delta to understand the evolution of software metrics [13]. They define code churn as the amount of change of a metric (for example the amount of source code lines added, modified, or deleted) and code delta as the difference of a metric between two revisions of a system, respectively. Studying the evolution of these parameters over time gives insight into what parts of a system are growing or contracting (code delta), and the amount of change needed (code churn) to achieve the growth or contraction. Using Lines of Code (LoC) as the metric for code churn (as we will do for the remainder of this paper), a higher than normal code churn can be linked to faults, code smells, security, and maintenance problems [10, 27, 33].

Code churn is the tangible effect of changes to the source code files of a system as it evolves. Besides studying how the source code evolves, source code repository mining also provides an opportunity to study social aspects like code ownership where a low degree of ownership indicate problematic code $[2,8]$.

\section{METHOD}

We perform a retrospective longitudinal case study where we analyze the released versions of JabRef using SACC based on the rules of the intended layered architecture (cf. Fig. 1). We also mine the Git source code repository to ascertain the code churn (added and deleted LoC) and the contributors for each commit. Based on this data, we can determine the accumulated code churn and code ownership for each source code file for each version of the application. Using the results from the SACC, we group files depending on whether they contain architectural violations, are refactored, or are normal.

From our research questions, we formulate the following hypotheses together with their complementing null hypotheses. If there is a link between architectural violations and code churn we expect to be able to refute the null hypothesis of $H(R Q 1)$ but not for $H(R Q 2)$, i.e. files with violations differ from normal files and become more normal after refactoring. The same reasoning is applied for code ownership and $H(R Q 3)$ and $H(R Q 4)$, respectively.

$H(R Q 1)$ : There is a significant difference in code churn for files with violations compared to normal files within releases before introduction of architectural layers.

$H(R Q 2)$ : There is a significant difference in code churn for files that are refactored compared to normal files after introduction of architectural layers.

$H(R Q 3)$ : There is a significant difference in code ownership for files with violations compared to normal files before introduction of architectural layers.

$H(R Q 4)$ : There is a significant difference in code ownership for refactored files compared to normal files after introduction of architectural layers.

\subsection{Detection of Architectural Violations}

SACC works by mapping each source code entity (e.g., class in an object-oriented system) to a component of the intended architecture (in the JabRef case, one of the layers). The actual dependencies (as implemented) are then checked to see whether they conform to the intended dependencies of the architecture or not. The initial mapping is often done by experts, i.e., the developers or the architect 
of the actual system [22, 28]. In our case, this is not feasible for two reasons; we are not system experts and there are too many releases to map. We instead implement an algorithm that can perform this mapping automatically for any version of JabRef. We can then check each version of JabRef for violations.

The algorithm is based on the intended architecture of JabRef as found in the documentation (cf. Fig. 1), in the architectural tests, and in the layers pattern [5]. Its first step is to use the mapping in release 3.7 of JabRef. We use this release to determine the classes in the Model, Logic, and GUI packages respectively. We can then find the same classes in other versions using a simple name comparison However, while this mapping most likely performs fine for releases near 3.7, it is more unlikely to perform well for earlier versions. For these earlier versions, a large portion of classes would not be mapped and thus not checked. To avoid this problem, we rely on the rules defined in the tests and infer a mapping that minimizes the number of violations, starting with the bottom layer (Model) and then iterate. When no more classes can be mapped, we move on to the next layer. For example, if an unmapped class is used by a class mapped to the Model layer, the unmapped class would be mapped to the Model layer as it would otherwise create a violation (e.g., if mapped to Logic or GUI). After the mapping, we check the conformance of each class and if there is a violation, the class is marked. We finally map classes to the corresponding source code Java file, since we want violations on a file level.

We implemented this using our own tool based on the ASM Java byte code manipulation and analysis framework version $5.0^{2}$. To ensure that our tool work as expected, we compared the results to the same analysis performed in Visual JArchitect ${ }^{3}$ 5.0.0. implemented using CQLinq queries. We could not find any discrepancies, so we conclude that our tool is correct.

\subsection{Data Collection}

To answer the research questions, we need to identify subset of releases before and after the architectural layers were introduced. The files in these subsets form our two main groups, Pre and Post We want to compare files that either contains violations or are refactored files with normal files (cf. Fig. 2), so we divide the two main groups into two sub groups. Each file of each release is mapped to one of these two subgroups, i.e., flagged as either containing a violation or not. This means that each file as it transitions from one release to another can be in one of four states:

(1) The file contains violation in both past and future releases,

(2) The file contained violations in past, but not in future releases (i.e., the violation is removed),

(3) The file contained no violation in past, but future releases have violations (i.e., a violation is added),

(4) The file has no violations, neither in past nor future release.

If we consider $\mathrm{H}(\mathrm{RQ} 1)$ and $\mathrm{H}(\mathrm{RQ} 3)$, files with violations are files in States 1, 2, or 3, and normal files are in State 4. For H(RQ2) and $\mathrm{H}(\mathrm{RQ} 4)$ refactored files are in State 2, and normal files are in State 4.

A confounding factor is that some release might focus on some particular task, such as adding/removing/changing a particular set

\footnotetext{
$\overline{{ }^{2} \text { http://asm.ow2.org }}$

${ }^{3} \mathrm{http}: / /$ www.jarchitect.com
}
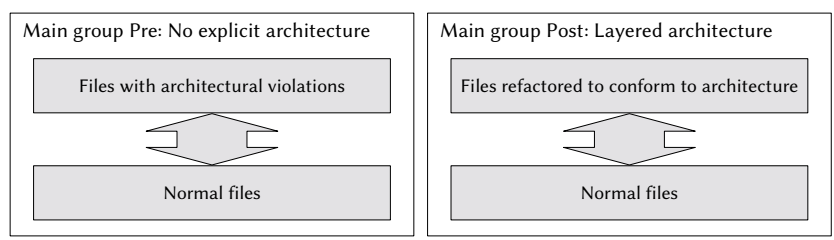

Figure 2: Overview of the collected data. Shows main groups and sub groups, arrows indicate hypothesis testing.

of features. This might cause a specific subset of files to be changed to a larger extent, which might skew the results. We address this problem by identifying an as large span of releases as possible for each main group. Another confounding factor to consider is the size of each file (in LoC). Since there are more LoC in larger files, they might also be more likely to have changes, violations, or other problems. To address this issue, we randomly select 100 files of the largest (highest average LoC) 200 files in each of the two main groups.

To test $\mathrm{H}(\mathrm{RQ} 3)$ and $\mathrm{H}(\mathrm{RQ} 4)$, we collect the number of unique contributors and the code churn for each contributor and file in the same subsets as we used for H(RQ1) and H(RQ2) (cf. Fig. 2).

The JabRef repository ${ }^{4}$ is mined using the git log command and we measure the size in LoC using cloc v.1.7.2 $2^{5}$.

\subsection{Data Analysis}

Statistical analysis is performed in R $3.3 .2^{6}$. The goal is to test if the null-hypothesis can be rejected. To test $H(R Q 1)$ and $H(R Q 2)$, we use the accumulated absolute code churn. We use this rather than relative measures since it is easier to interpret the effect size for absolute measures. To test $\mathrm{H}(\mathrm{RQ} 3)$ and $\mathrm{H}(\mathrm{RQ} 4)$ we compute the biased Gini coefficient based on the accumulated code churn for each contributor to a source code file. The Gini coefficient is a measure of inequality ranging from 0.0 to 1.0 , where 1.0 is the most unequal $[12,37]$. For example, if there is a file that has seven unique developers that contribute to it, the Gini coefficient gives insight into the distribution of code churn between the contributors, i.e., did they all contribute equally much or not. So, the Gini coefficient allows us to determine if there is a clear code ownership of a file or not. The unbiased Gini coefficient will move towards 1.0 (in a perfect equal distribution) as the number of contributors increase, so we use a bias factor is based on the number of contributors to ensure that the value range of the Gini coefficient is between 0.0 and 1.0.

We assume that neither the churn data nor the Gini coefficient are normally distributed for this data set, so we use the non-parametric Wilcoxon Rank Sum test to perform the tests of our hypothesis. We use $p<0.05$ to indicate significance. We will perform two tests per main groups and the p-value will not need adjustment.

\section{RESULTS}

We run our automatic SACC algorithm on all released versions of JabRef, and find that it performs as expected. Initially, the number of

\footnotetext{
${ }^{4}$ https://github.com/JabRef/jabref

${ }^{5}$ https://github.com/AlDanial/cloc

${ }^{6}$ https://www.r-project.org
} 
Table 1: Comparison of LoC in files per group.

\begin{tabular}{lrrrr}
\hline & $\begin{array}{c}\text { Pre } \\
\text { Normal }\end{array}$ & $\begin{array}{c}\text { Pre } \\
\text { Violations }\end{array}$ & $\begin{array}{c}\text { Post } \\
\text { Normal }\end{array}$ & $\begin{array}{c}\text { Post } \\
\text { Refactored }\end{array}$ \\
\hline \#Files & 67 & 33 & 80 & 20 \\
Median & 243 & 265 & 205 & 184 \\
Average & 335 & 346 & 222 & 225 \\
Min & 116 & 115 & 82 & 128 \\
Max & 1258 & 1142 & 495 & 476 \\
SD & 252 & 251 & 82 & 103 \\
\hline
\end{tabular}

Table 2: Results of hypothesis testing, shows the resulting pvalue, $r$-value, $95 \%$ confidence interval, and the median difference.

\begin{tabular}{lccrr}
\hline Hypothesis & p & \multicolumn{1}{c}{ r } & \multicolumn{1}{c}{ conf. int. } & med. diff. \\
\hline H(RQ1) & 0.016 & 0.241 & $79-1013$ & -844 \\
H(RQ2) & 0.069 & 0.180 & $-52-1472$ & -737 \\
H(RQ3) & 0.827 & 0.022 & $-0.057-0.07$ & -0.001 \\
H(RQ4) & 0.301 & 0.104 & $-0.079-0.023$ & 0.027 \\
\hline
\end{tabular}

files with violations steadily grows as the system itself grows. This trend is reversed starting with release 2.11, but it takes until some releases after version 3.0 for a majority of files to be refactored.

We select version 2.11 as the version where the architectural refactoring into a layered architecture was initiated. In this release, the number of actual violations start to decline and the Logic package is introduced. Version 3.0 was released only 18 days after 2.11 The subset of releases after the new architecture is introduced (Post) thus consists of the 12 releases from 2.11 to 3.8.2. We use the same number of releases to capture roughly the same amount of work in the releases prior to introduction of the new architecture. This makes release $2.9 \mathrm{~b} 2$ the first release to be included in the Pre subset.

A Pearson Correlation test between code churn and LoC show a moderate but significant correlation. There is a tendency for large files to have a higher code churn $(p<0.05, r=0.58,95 \%$ confidence interval: $0.55-0.61$ ).

We removed one file with violations from the data as this was considered an outlier (Util. java). The file had a churn of 33,189 while the second most churned file in the Pre group had a churn of 11,183 . We then select the 100 files that form the sub groups. Table 1 provides an overview of the $\mathrm{LoC}$ in the groups and Figure 3 shows a box plot for the groups.

We use the Wilcoxon Rank Sum test to determine if we can reject the null hypothesis at $p<0.05$ for our hypotheses. Figure 4 shows the code churn for each sub group. For H(RQ1), we can reject the null hypothesis and find a significant difference in code churn. For $\mathrm{H}(\mathrm{RQ2})$, we cannot reject the null hypothesis (cf. Table 2). Figure 5 shows the Gini coefficient for each group. As the box plot suggests, there is no difference within the groups. We cannot reject the null hypothesis for $\mathrm{H}(\mathrm{RQ} 3)$ or $\mathrm{H}(\mathrm{RQ} 4)$ (cf. Table 2).

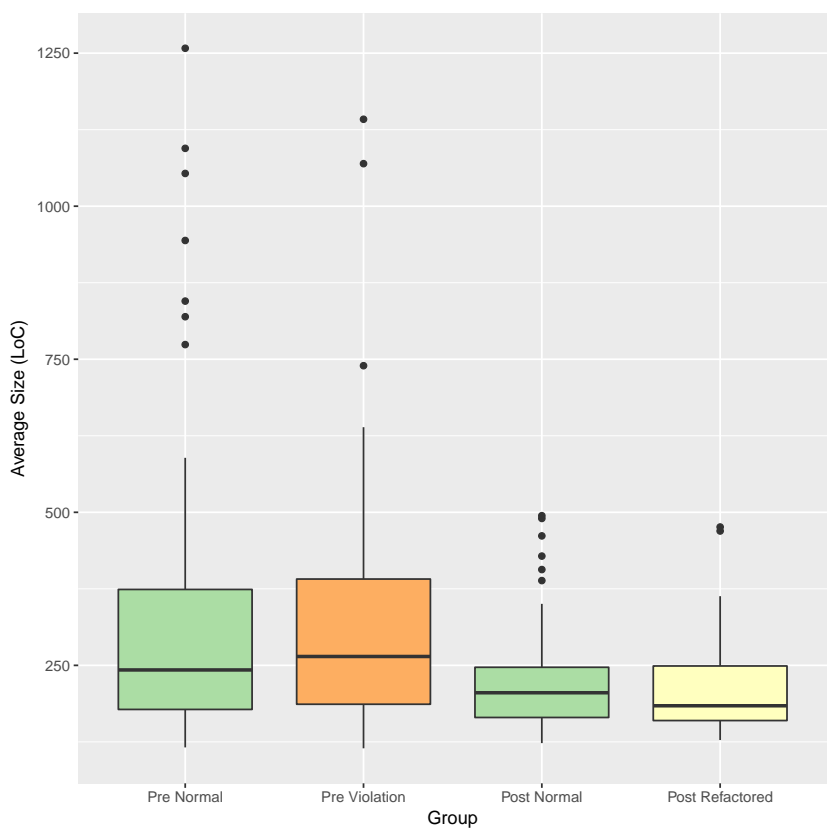

Figure 3: Average Size (LoC) in the four groups.

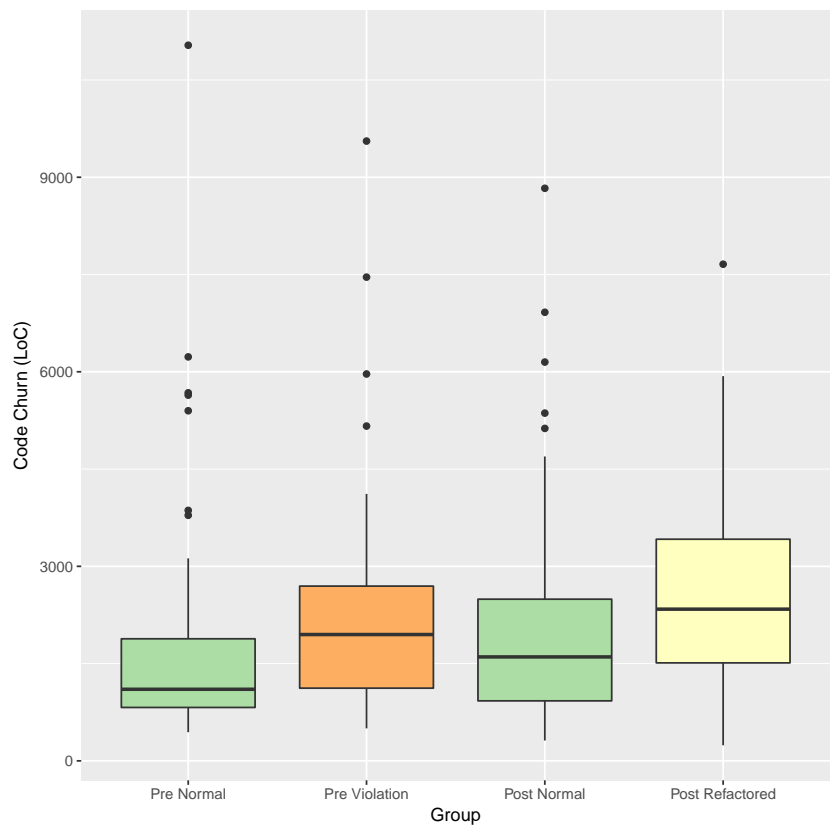

Figure 4: Code Churn in the four groups. Normal files are compared to files with violations in the Pre group. Normal files are compared to refactored files in the Post group

\section{RELATED WORK}

In [25], a Dependency Structure Matrix is used to form two metrics of modularity based on coupling. The metrics are used to analyze 19 versions, from early 1998 to early 2000, of Mozilla in a longitudinal 


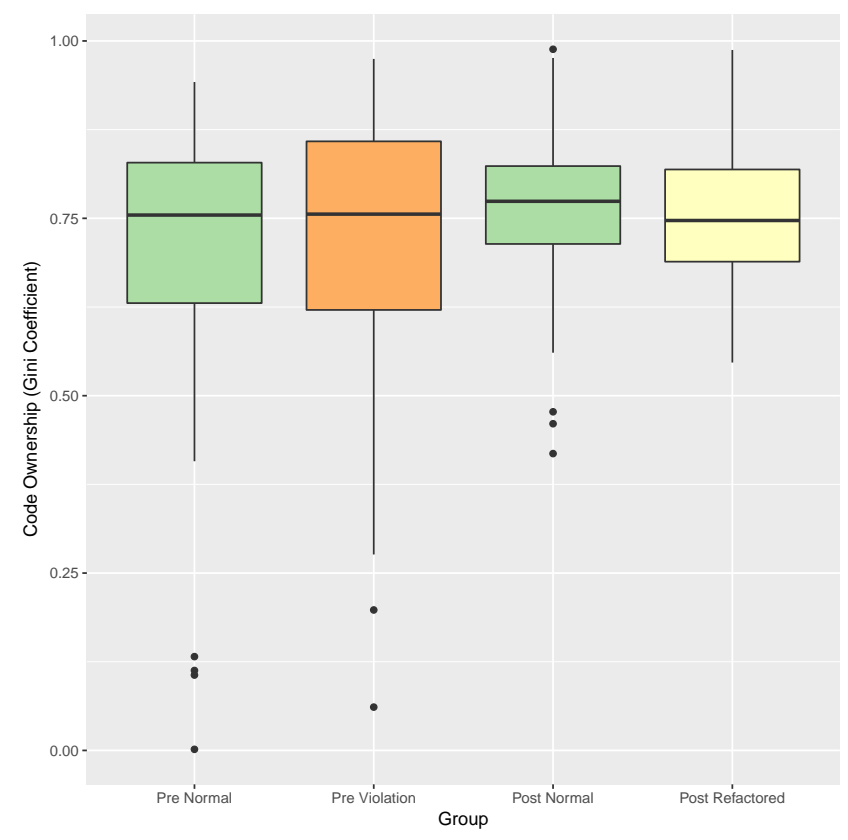

Figure 5: Code ownership in the four groups based on the Gini Coefficient. Normal files are compared to files with violations in the Pre group. Normal files are compared to refactored files in the Post group

study during an infamous redesign effort where the all of the old source code was scrapped [38]. In the study, the redesign had a positive impact on both metrics. The focus in [25] is, however, not on architectural violations but rather on managerial impact on system architecture. In [11] Mozilla's architecture is again analyzed, this time version M9, released August 1999. The conclusion from this analysis is that the architecture has either deteriorated very rapidly or that architecture was not a focal point from the onset in the project. MacCormack et al. do not find this surprising, as the development was under extreme pressure during the "browser wars". However, this study also does not focus on violations of the architecture per se, but rather at reverse engineering tools and increasing tool interoperability.

In [7], a large dataset of source code $(100,000,000 \mathrm{LoC}, 50 \mathrm{sub}-$ systems and 5,000 modules) and complete change management information for 15 years of releases is investigated to determine whether the developer's notions of code decay is real. The study analyses a single subsystem (100 modules) and conclude that the code has indeed decayed in relation to changes made to it. There was however no evidence of dramatic or widespread decay. The study is focused on change management data (file updates, additions, deletions) but not on code churn or architectural violations.

An experiment by Knodel et al. [20,21] indicate that an effect of architectural violations cause unneeded effort when evolving software systems. The setup is an experiment with two versions of the same professionally developed system; one with violations and one without. The same evolutionary task is replicated three tims and is performed by randomized development teams consisting of both professional developers and students. The experiment showed that it was harder both in terms of effort and correctness to evolve the eroded version of the system, though the evolutionary task selected of course is important [20].

In [3] refactorings are guided by architectural violations. An improvement in code metrics is reported and developers found it easier to evolve the system after refactoring. However, no quantitative data is used to show this.

In [32] the architecture of a large scale (25 MLoC) business application is refactored into a layered and more modular architecture. They report on a set of "home-grown gate keeper tools" that check conformance to layer and module rules. They report on a set of metrics and improvements of these during the two-year effort of refactoring parts of the system into the new architecture.

In [9], Faragó et al. find that code that has been heavily modified in the past tend to predict a decrease maintainability in future modifications. They compare the average of the cumulative code churn (the number of added and removed lines up to a certain point in time) for each file with the maintainability change (positive or negative) for each commit. The maintainability is computed as an aggregation of code metrics. They investigate four cases and find a significant relation. This research is especially interesting as it establishes a cause and effect relation, i.e., they use the historical code churn of the files and estimate the effect of a future commit on maintainability.

Churn- and architectural dependency-metrics are combined in [27] to improve prediction of post-release component failures in Windows Server 2003. The code churn measures collected for each component is the sum of the number of lines added, deleted or modified, as well as the number of files touched and the number of changes made (i.e., the number of commits). Using this information in combination with architectural dependencies they build a multiple linear regression model to predict failures post release.

Faragó et al. [8] has also investigated code ownership effect on maintainability in four systems. The measure of maintainability is an aggregation of source code metrics. The measure of code ownership is calculated for each commit and is based on the geometric mean of the accumulated number of different contributors for each file in the commit. This means that the ownership value is based on the files included in the commit. They find a statistically significant relation between a high degree of code ownership and a positive maintainability trend in three of the four cases. Their conclusion is that common code is more likely to accumulate maintenance problems than code with more clear authorship.

Code ownership has also been studied in the large OSS Apache server [26]. It is analyzed in order to find signs of code ownership on file and module-level. Mockus et al. investigate how many developers contribute to each file, with a cut off at $10 \%$ to remove very small contributions. They find that there is no code ownership at file or module level, and that the "core team contribute to various modules as needed". They also put forth the hypothesis that: "For projects that are so large that 10-15 developers cannot write $80 \%$ of the code in a reasonable time frame, a strict code ownership policy will have to be adopted to separate the work of additional groups, creating, in effect, several related OSS projects.". 


\section{DISCUSSION}

We found that the amount of change needed (code churn) to files in JabRef that contain architectural violations is often larger than the amount of change needed to files that do not contain architectural violations, if there is a lack of explicit architecture. The size of this effect is medium $(r=0.241$, median difference $=-844)$, i.e., there is an expectation that a file containing violations require more churn than a file that does not. After the architectural refactoring effort, files that are refactored show a more normal change pattern when compared to normal files for the same time period. In this latter case, it should be noted that the actual refactoring is also included and that there could be some time before the file is actually refactored. This could explain why there still seems to be a difference in churn post refactoring (median difference $=-737$ ). It would be interesting to remove the effect of refactoring itself from the analysis. This would, however, require another type of data analysis as different files are refactored at different points in time so a direct comparison of code churn would not be possible. In general, our findings indicate that a tangible effect of architectural violations in source code files is higher code churn compared to normal files. Refactoring to achieve architectural compliance seems to make the code churn of refactored files comparable to normal files. However, there is no implied causality in our analysis, violations might be added because the code is changed a lot or violations drive code churn (i.e., because of these dependencies we need to make changes).

We could not find any difference in code ownership in any of the groups. In general, this is could be regarded as contrary to the effects found in Faragó et al. [8], i.e., we should be able to reject the null hypothesis of $\mathrm{H}(\mathrm{RQ} 3)$. However, there are two major differences. First, we compare to architectural violations and not maintenance as measured by code metrics. Second, we measure ownership differently as we are interested in a general difference between groups while Faragó et al. [8] is interested the accumulated ownership of files in each commit. However, in studying the OSS Apache server, no real code ownership evolved [26]. Our findings support their hypothesis, JabRef is not big or complex enough to need a strict code ownership policy. There could also be team effects, i.e., a few developers working closer together, something that is not visible in our analysis.

We focus on the direct effect of violations, i.e., added responsibility in the file that contain the violations. In some cases, it may be that the violation (e.g., an added attribute) is actually used in other files, i.e., the effect of the violation is not isolated to a single file. It could thus be interesting to combine coupling and code churn as done in [2] and [27].

Before the new architecture was introduced, there was no documented notion of structure that could consciously be violated. Our analysis is rather based on determining the impact of a new architecture on the existing source code. There could be other architectures that would have a larger impact. It should be possible to combine SACC and repository mining to better understand the importance of architectural compliance and the effects of an architectural refactoring. One approach could be to use repository data to determine the effects of a proposed architecture on the source code. This could determine whether the rules proposed by the architecture can actually be used to identify files that are known to be problematic (for example by measuring code churn), and find trade-offs between major refactorings and possible effect. If no such effect can be found there is no data to support the refactoring effort. This would, in essence, be a way to use SACC to support data driven agile architecting.

\section{THREATS TO VALIDITY}

We only investigate one case in a retrospective study so our findings are hard to generalize. More cases and studies regarding architectural violations and code churn in similar but different contexts are needed to confirm or refute our findings. However, it is generally not easy to identify suitable subjects that use SACC. Also, in some cases found violations are not removed [3, 31]. In OSS, JabRef could be a unique example of using SACC to rigidly define the architecture, and as such it is worth studying.

There are possible errors in our measurements. Our algorithm to map Java classes to architectural components is conservative as it tries to minimize violations. However, the name-based mapping accounts for the majority of mapping, and this should be correct as per the developers' mapping. We count each LoC as equal, which can be a problem, since in practice, one line might be the result of much effort or one file may encapsulate some really complicated lines. Such differences are not visible in our analysis. This is a general problem in source code metrics and it is important to realize that metrics are an abstraction that do not show every detail in every situation.

Another potential problem in our analysis is subject independence, i.e., a file is not an independent subject and churn in one file may affect churn in other files. We address this by using a large set of commits and releases, and random sampling.

\section{CONCLUSION AND FUTURE WORK}

In JabRef we could use SACC to find a significant difference in code churn between files that have architectural violations compared to normal files before a new architecture is introduced (RQ1). Code churn for files that contain violations becomes more normal when these files are refactored to remove the violations (RQ2). We could not find any difference in code ownership for files with architectural violations compared to normal files, neither before nor after the new architecture was introduced (RQ3 and RQ4). We conclude that in our case, architectural violations and code churn are linked, but not code ownership. Non-normal code churn can be a tangible, measurable, effect of architectural violations in source code. However, more research is needed regarding this link; if violations are the cause, the size of the effect, the cost of refactoring, and whether it is generalizable to other systems, are examples of what needs to be further investigated.

We will continue our work in combining SACC and repository data mining by studying and evaluating simple and efficient methods for SACC in evolving systems. As a next step, we plan to investigate the more direct effect of refactoring files in JabRef, possibly extending this to include different type or amounts of violations and whether there is an effect on classes that depend on these violations. We also plan to work on visualizations of architectural evolution and SACC, and combine this for efficient semi-automatic code to architecture mapping techniques. 


\section{REFERENCES}

[1] Nour Ali, Sean Baker, Ross O'Crowley, Sebastian Herold, and Jim Buckley. 2017. Architecture consistency: State of the practice, challenges and requirements. Empirical Software Engineering (2017), 1-35. https://doi.org/10.1007/ s10664-017-9515-3

[2] Christian Bird, Nachiappan Nagappan, Brendan Murphy, Harald Gall, and Premkumar Devanbu. 2011. Don't touch my code!: examining the effects of ownership on software quality. In Proceedings of the 19th ACM SIGSOFT symposium and the 13th European conference on Foundations of software engineering. ACM, 4-14.

[3] Fabrice Bourquin and Rudolf K Keller. 2007. High-impact refactoring based on architecture violations. In 11th European Conference on Software Maintenance and Reengineering (CSMR). IEEE, 149-158.

[4] Jim Buckley, Nour Ali, Michael English, Jacek Rosik, and Sebastian Herold. 2015 Real-Time Reflexion Modelling in architecture reconciliation: A multi case study. Information and Software Technology 61 (2015), 107-123.

[5] Frank Buschmann, Kelvin Henney, and Douglas Schimdt. 2007. Pattern - Oriented Software Architecture: On Patterns and Pattern Language. Vol. 5. John Wiley \& Sons.

[6] Lakshitha De Silva and Dharini Balasubramaniam. 2012. Controlling software architecture erosion: A survey. Journal of Systems and Software 85, 1 (2012), $132-151$.

[7] Stephen G Eick, Todd L Graves, Alan F Karr, James S Marron, and Audris Mockus 2001. Does code decay? assessing the evidence from change management data. IEEE Transactions on Software Engineering 27, 1 (2001), 1-12.

[8] Csaba Faragó, Péter Hegedűs, and Rudolf Ferenc. 2015. Code ownership: Impact on maintainability. (2015).

[9] Csaba Faragó, Péter Hegedủs, and Rudolf Ferenc. 2015. Cumulative code churn Impact on maintainability. In Source Code Analysis and Manipulation (SCAM), 2015 IEEE 15th International Working Conference on. IEEE, 141-150.

[10] Csaba Faragó, Péter Hegedus, Gergely Ladányi, and Rudolf Ferenc. 2015. Impact of version history metrics on maintainability. In Advanced Software Engineering \& Its Applications (ASEA), 2015 8th International Conference on. IEEE, 30-35.

[11] Michael W Godfrey and Eric HS Lee. 2000. Secrets from the monster: Extracting MozillaâĂŹs software architecture. In Proceedings of Second Symposium on Constructing Software Engineering Tools (CoSETâ̆̌́O0).

[12] Olga Goloshchapova and Markus Lumpe. 2013. On the application of inequality indices in comparative software analysis. In Software Engineering Conference (ASWEC), 2013 22nd Australian. IEEE, 117-126.

[13] Gregory A Hall and John C Munson. 2000. Software evolution: code delta and code churn. Journal of Systems and Software 54, 2 (2000), 111-118.

[14] Ahmed E Hassan. 2008. The road ahead for mining software repositories. In Frontiers of Software Maintenance, 2008. FoSM 2008. IEEE, 48-57.

[15] Sebastian Herold, Martin Blom, and Jim Buckley. 2016. Evidence in architecture degradation and consistency checking research: preliminary results from a literature review. In Proccedings of the 10th European Conference on Software Architecture Workshops. ACM, 20

[16] Sebastian Herold and Jim Buckley. 2015. Feature-Oriented Reflexion Modelling. In Proceedings of the 2015 European Conference on Software Architecture Workshops. ACM, 59.

[17] Lorin Hochstein and Mikael Lindvall. 2005. Combating architectural degeneration: a survey. Information and Software Technology 47, 10 (2005), 643-656.

[18] Sunny Huynh, Yuanfang Cai, Yuanyuan Song, and Kevin Sullivan. 2008. Automatic modularity conformance checking. In ACM/IEEE 30th International Conference on Software Engineering. IEEE, 411-420.

[19] Eirini Kalliamvakou, Georgios Gousios, Kelly Blincoe, Leif Singer, Daniel M German, and Daniela Damian. 2014. The promises and perils of mining GitHub. In Proceedings of the 11th working conference on mining software repositories. ACM, 92-101.

[20] Jens Knodel. 2011. Sustainable Structures in Software Implementations by Live Compliance Checking. Fraunhofer Verlag.

[21] Jens Knodel, Mikael Lindvall, Dirk Muthig, and Matthias Naab. 2006. Static evaluation of software architectures. In 10th European Conference onSoftware Maintenance and Reengineering (CSMR). IEEE, 10-pp.

[22] Jens Knodel and Daniel Popescu. 2007. A comparison of static architecture compliance checking approaches. In The Working IEEE/IFIP Conference on Software Architecture. IEEE, 12-12.

[23] Rainer Koschke. 2013. Incremental reflexion analysis. fournal of Software: Evolution and Process 25, 6 (2013), 601-637.

[24] Rainer Koschke and Daniel Simon. 2003. Hierarchical Reflexion Models. In 10th Working Conference on Reverse Engineering. IEEE, 36.

[25] Alan MacCormack, John Rusnak, and Carliss Y Baldwin. 2006. Exploring the structure of complex software designs: An empirical study of open source and proprietary code. Management Science 52, 7 (2006), 1015-1030.

[26] Audris Mockus, Roy T Fielding, and James Herbsleb. 2000. A case study of open source software development: the Apache server. In Software Engineering, 2000. Proceedings of the 2000 International Conference on. IEEE, 263-272.
[27] Nachiappan Nagappan and Thomas Ball. 2007. Using software dependencies and churn metrics to predict field failures: An empirical case study. In Empirical Software Engineering and Measurement, 2007. ESEM 2007. First International Symposium on. IEEE, 364-373.

[28] Leonardo Passos, Ricardo Terra, Marco Tulio Valente, Renato Diniz, and Nabor das Chagas Mendonca. 2010. Static architecture-conformance checking: An illustrative overview. IEEE software 27, 5 (2010), 82.

[29] André Postma. 2003. A method for module architecture verification and its application on a large component-based system. Information and Software Technology 45, 4 (2003), 171-194.

[30] Leo Pruijt, Christian Koppe, and Sjaak Brinkkemper. 2013. Architecture compliance checking of semantically rich modular architectures: A comparative study of tool support. In International Conference on Software Maintenance (ICSM). IEEE, 220-229.

[31] Jacek Rosik, Andrew Le Gear, Jim Buckley, Muhammad Ali Babar, and Dave Connolly. 2011. Assessing architectural drift in commercial software development: a case study. Software: Practice and Experience 41, 1 (2011), 63-86.

[32] Santonu Sarkar, Shubha Ramachandran, G Sathish Kumar, Madhu K Iyengar, K Rangarajan, and Saravanan Sivagnanam. 2009. Modularization of a large-scale business application: A case study. IEEE software 26, 2 (2009), 28-35.

[33] Yonghee Shin, Andrew Meneely, Laurie Williams, and Jason A Osborne. 2011. Evaluating complexity, code churn, and developer activity metrics as indicators of software vulnerabilities. IEEE Transactions on Software Engineering 37, 6 (2011), 772-787.

[34] Ricardo Terra and Marco Tulio Valente. 2009. A dependency constraint language to manage object-oriented software architectures. Software: Practice and Experience 39, 12 (2009), 1073-1094.

[35] Jilles Van Gurp and Jan Bosch. 2002. Design erosion: problems and causes. fournal of systems and software 61, 2 (2002), 105-119.

[36] Jilles Van Gurp, Jan Bosch, and Sjaak Brinkkemper. 2003. Design Erosion in Evolving Software Products. In ELISA workshop. 134.

[37] Rajesh Vasa, Markus Lumpe, Philip Branch, and Oscar Nierstrasz. 2009. Comparative analysis of evolving software systems using the Gini coefficient. In Software Maintenance, 2009. ICSM 2009. IEEE International Conference on. IEEE, 179-188.

[38] Wikipedia. 2016. History of Mozilla Application Suite - Wikipedia, The Free Encyclopedia. (2016). en.wikipedia.org/wiki/History_of_Mozilla_Application_ Suite [Online; accessed 07-Jan-2017].

[39] Tao Xie, Suresh Thummalapenta, David Lo, and Chao Liu. 2009. Data mining for software engineering. Computer 42, 8 (2009). 